\title{
Reconstructing the Scene: New Views of Supernovae and Progenitors from the SNSPOL Project
}

\author{
Jennifer L. Hoffman ${ }^{1}$, G. Grant Williams ${ }^{2,3}$, Douglas C. Leonard ${ }^{4}$, \\ Christopher Bilinski ${ }^{3}$, Luc Dessart ${ }^{5}$, Leah N. Huk ${ }^{1}$, \\ Jon C. Mauerhan ${ }^{6}$, Peter Milne ${ }^{3}$, Amber L. Porter ${ }^{7}$, \\ Nathan Smith ${ }^{3}$ and Paul S. Smith ${ }^{3}$ \\ ${ }^{1}$ Department of Physics \& Astronomy, University of Denver, \\ 2112 E. Wesley Ave., Denver, CO 80210-6900, USA \\ email: jennifer.hoffman@du.edu \\ ${ }^{2}$ MMT Observatory, P.O. Box 210065, \\ University of Arizona, Tucson, AZ 85721-0065, USA \\ ${ }^{3}$ Steward Observatory, University of Arizona, \\ 933 N. Cherry Avenue, Tucson, AZ 85721, USA \\ ${ }^{4}$ Department of Astronomy, San Diego State University, \\ 5500 Campanile Drive, San Diego, CA 92182-1221, USA \\ ${ }^{5}$ Unidad Mixta Internacional Franco-Chilena de Astronomía (CNRS UMI 3386), \\ Departamento de Astronomía, Universidad de Chile, \\ Camino El Observatorio 1515, Las Condes, Santiago, Chile \\ ${ }^{6}$ Department of Astronomy, University of California, \\ Berkeley, CA 94720-3411, USA \\ ${ }^{7}$ Department of Physics and Astronomy, Clemson University, \\ 118 Kinard Laboratory, Clemson, SC 29634
}

\begin{abstract}
Because polarization encodes geometrical information about unresolved scattering regions, it provides a unique tool for analyzing the 3-D structures of supernovae ( $\mathrm{SNe}$ ) and their surroundings. SNe of all types exhibit time-dependent spectropolarimetric signatures produced primarily by electron scattering. These signatures reveal physical phenomena such as complex velocity structures, changing illumination patterns, and asymmetric morphologies within the ejecta and surrounding material. Interpreting changes in polarization over time yields unprecedentedly detailed information about supernovae, their progenitors, and their evolution.

Begun in 2012, the SNSPOL Project continues to amass the largest database of time-dependent spectropolarimetric data on SNe. I present an overview of the project and its recent results. In the future, combining such data with interpretive radiative transfer models will further constrain explosion mechanisms and processes that shape SN ejecta, uncover new relationships among SN types, and probe the properties of progenitor winds and circumstellar material.
\end{abstract}

Keywords. polarization, astronomical databases:miscellaneous, supernovae: general

\section{Supernovae and polarization}

Because supernovae ( $\mathrm{SNe}$ ) can be seen at great distances, they provide clues about stellar evolution in faraway galaxies and in the cosmological past. SNe are broadly classified into "thermonuclear" and "core-collapse" categories. Thermonuclear (Type Ia) supernovae likely arise from binary stars in which a white dwarf accretes material from its companion and becomes unstable (although white-dwarf mergers may be a viable mechanism for some SNe Ia; see, e.g., Ruiz-Lapuente 2014). Core-collapse supernovae (CCSNe; 
Types Ib/c and II) are thought to arise from massive stars that exhaust the fuel in their cores and undergo catastrophic collapse and subsequent neutrino-driven explosion. The wide range of different subtypes of CCSNe is not well understood, but seems to be related to mass loss in late stages of evolution, which affects the amount of circumstellar material (CSM) surrounding the doomed progenitor star (e.g., Smart 2009).

All types of SNe display intrinsic polarization signatures that vary over time, but the behavior of this polarization differs among subtypes (Wang \& Wheeler 2008). SN polarization is caused primarily by light scattering from free electrons in ionized ejecta material. An unresolved spherical scattering region yields no net polarization, but an elongated or otherwise aspherical electron-scattering region produces a net wavelengthindependent polarization signal. Thus, the simplest case of SN polarization is a constant continuum signal that reveals the presence of aspherical ejecta (Höflich 1991). Tracing this continuum polarization over time as the SN evolves can reveal the complex nature of the surrounding material. For example, SN 2004dj and related objects have very low continuum polarization at early times but show a polarization spike at the end of the plateau phase, revealing an inner, aspherical core as the the spherical outer envelope becomes transparent (Leonard et al. 2006; Chornock et al. 2010; Dessart \& Hillier 2011).

More complex wavelength-dependent scenarios occur when clumps or inhomogeneities within the ejecta obscure portions of the scattering region (Kasen et al. 2003), when circumstellar material surrounds the ejecta and contributes its own scattering signal (Hoffman et al. 2008), or when line photons interact differently with the ejecta than do continuum photons. Combinations of these effects are common. The time domain adds an additional dimension of complication, as the relative contributions of each of these effects may change as the SN evolves. (However, the constant contribution of interstellar polarization [ISP] can be constrained with time-series observations, e.g., Leonard et al. 2000; Hoffman et al. 2008.) The vast majority of SNe studied with more than one epoch of polarimetry have displayed variability over time in the continuum, lines, or both.

Time-dependent spectropolarimetry thus provides us with an unprecedentedly detailed view into the complex and variable structures surrounding $\mathrm{SNe}$ of all types. This allows us to probe not only the SN mechanism itself, but also to constrain progenitor properties by measuring the characteristics (density, composition, velocity, geometrical structure) of the stellar winds and eruptions that preceded the final explosion.

\section{The SNSPOL Project}

Begun in 2012 and funded by the National Science Foundation, the SNSPOL Project (PI: G.G. Williams) continues to amass the largest database of time-dependent spectropolarimetric data on supernovae of all types. The SNSPOL Project obtains observations of bright SNe roughly once per month using the CCD Imaging/Spectropolarimeter (SPOL; Schmidt, Elston, \& Lupie 1992) at either the 61" Kuiper telescope on Mt. Bigelow, the 2.3-m Bok on Kitt Peak, or the 6.5-m MMT on Mt. Hopkins. Supporting observations include optical and near-IR photometry from the Mount Laguna Observatory (Khandrika et al. 2014) and ISP probe star observations from the HPOL spectropolarimeter at the University of Toledo's Ritter Observatory (Davidson et al. 2014). To date, the SNSPOL project has obtained spectropolarimetric data for over $80 \mathrm{SNe}$, of which 59 have been observed at more than one observational epoch.

SNSPOL's monthly observation cadence allows us to monitor the spectropolarimetric evolution of our targets over their optically visible lifetimes in more detail than has previously been possible. In the sections below, we review some of the most significant results from the SNSPOL Project to date. 

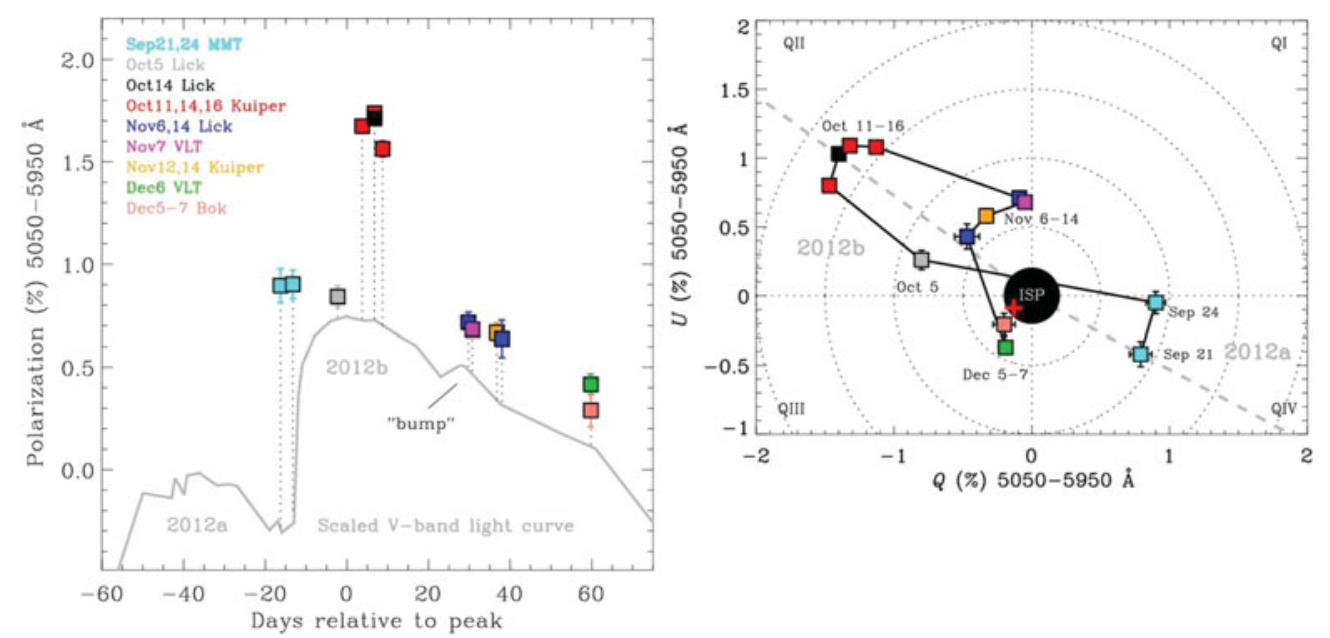

Figure 1. Left: Temporal evolution of the total $V$-band polarization for SN 2009ip in 2012. Filled squares represent polarization measurements; the gray line represents the $V$-band light curve. Right: Filled squares represent the same polarization measurements as at left, now plotted in the $Q-U$ plane. ISP is constrained to be less than $0.2 \%$, illustrated by the black dot. Dashed lines represent the position angles $166^{\circ}$ and $72^{\circ}$, as measured at the first epoch on Sep. 21 and peak polarization on Oct. 14, respectively. The approximate point-reflection symmetry between 2012a and the peak of 2012b suggests two separate and roughly orthogonal components of polarization on the sky. Figure from Mauerhan et al. (2014), courtesy Oxford University Press and the Royal Astronomical Society. For the full-color figure, see that paper.

\subsection{SN 2009ip}

SNSPOL observations of the peculiar transient SN 2009ip provided new insights into the nature of its two 2012 brightness peaks (Fraser et al. 2013; Mauerhan et al. 2013; Levesque et al. 2014; Margutti et al. 2014). SNSPOL data showed that these two peaks possessed orthogonal polarization position angles (Fig. 1; Mauerhan et al. 2014). This suggests that two distinct scattering regions produced the polarization at these two epochs. We concluded that the 2012a peak arose from the maximum brightness of a true core-collapse SN explosion and the corresponding polarization was due to asphericity of the SN ejecta (Mauerhan et al. 2014). The 2012b peak was likely due to CSM interaction, and its polarization angle suggests this CSM was equatorially concentrated orthogonal to the ejecta axis. This geometry is similar to the one suggested by Levesque et al. (2014) based on spectral modeling. This CSM likely arose from the progenitor's mass loss in late evolution, and its flattened nature could indicate a binary origin.

\section{2. $S N$ 2011dh}

In the case of the Type IIb supernova SN 2011dh, we combined four SNSPOL observations with additional spectropolarimetry from the Lick and Palomar Observatories. The commonalities in position angle between the early continuum polarization and that of the $\mathrm{H} \alpha$ and $\mathrm{He} \mathrm{I}$ lines led us to favor a picture in which fast-rising plumes of radioactive ${ }^{56} \mathrm{Ni}$ produced clumpy excitation of the SN envelope (Mauerhan et al. 2015; Fig. 2). This scenario may link SN 2011dh with other SN types and with SNRs such as Cas A.

\section{3. $S N 2014 J$}

Seven SNSPOL observations of the nearby Type Ia SN 2014J revealed that this object displayed typical polarization behavior for a SN Ia, with nearly spherical ejecta combined with a clumpy distribution of silicon; circumstellar dust may also contribute to the 


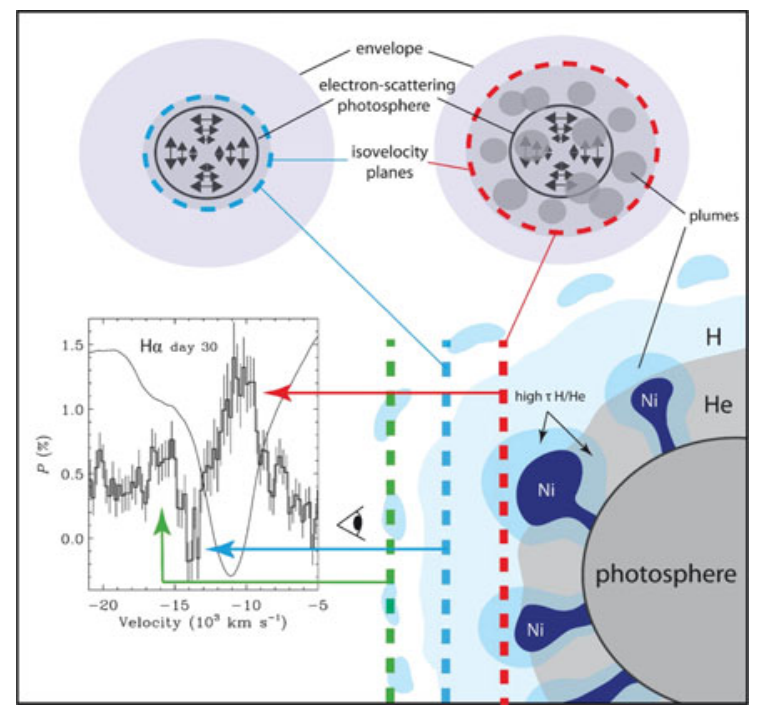

Figure 2. Illustration of the formation of the polarized $\mathrm{H} \alpha$ line in SN 2011dh. The upper diagrams are face-on views; the lower diagram illustrates the transverse view. Several isovelocity planes (dashed lines) are depicted along with their predicted polarization characteristics and locations within the polarized line profile. Figure from Mauerhan et al. (2015), courtesy Oxford University Press and the Royal Astronomical Society. For the full-color figure, see that paper.

continuum polarization in this object (Porter et al. 2016). Our spectropolarimetric data also allowed us to investigate the nature of the ISP in the host galaxy, M82. The dust grains along this sightline in M82 are smaller than those making up typical Milky Way dust.

\subsection{SN 2012au}

The polarization of SN 2012au evolved from a large magnitude of $\sim 2 \%$ at early times to nearly zero within 3 months, while its well-defined polarization angle gradually disappeared (Hoffman et al. 2014; Fig. 3). Rather than a dramatic change in large-scale ejecta geometry, we attribute this behavior to a receding of the photosphere through an outer region with greater asymmetry to an inner region with greater symmetry (the opposite behavior from SN 2004dj; Leonard et al. 2000). The He I $\lambda 5876$ emission line displayed complex intrinsic polarization behavior with a position angle nearly $90^{\circ}$ different from that of the continuum, a possible disk-jet signature. Analysis of this SN is still underway.

\section{The future of SNSPOL}

As the SNSPOL Project continues to collect data on a broad variety of SNe, our focus will shift from observing as many objects as possible to improving wavelength and time coverage for particular targets of interest. We aim to improve the statistical sample size of the various SN types studied with spectropolarimetry; this will allow us to move from analyzing individual SNe to considering the relationships among SN subtypes and among different events within a given subtype. These larger-scale studies will allow us to draw connections between SNe and their progenitors more clearly than ever before.

Our collaboration is also developing radiative transfer modeling capabilities that include complex polarization effects (Dessart \& Hillier 2011; Huk et al. 2017, this volume). These will allow us to better interpret the spectropolarimetric data obtained by the 

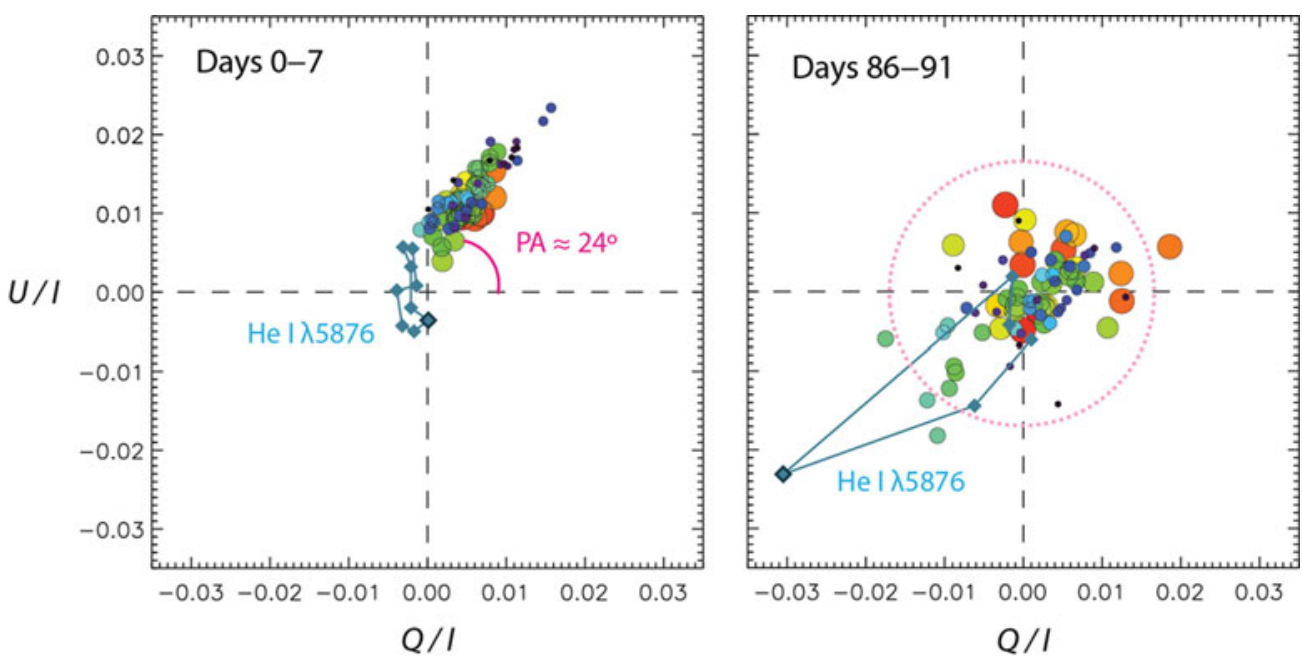

Figure 3. Two epochs of polarization of the Type Ib SN 2012au from SNSPOL. Wavelength points (binned to $50 \AA$ ) run from small to large circles; the connected diamond points represent the He I $\lambda 5876$ emission line, binned to $10-20 \AA$. The continuum data show a clear polarization axis at $\sim 24^{\circ}$ at early times, but scatter around 0 at later times (suggesting a low ISP contribution; dotted circle). The helium line is intrinsically polarized differently from the continuum at all times. Figure from Hoffman et al. (2014).

SNSPOL Project. The confrontation of observations with models will ramp up in the near future as the project shifts its focus from data collection to larger-scale analysis.

Acknowledgments. JLH and LNH thank the IAU for generous travel support. The SNSPOL Project is supported by NSF awards AST-1210599, AST-1210311, and AST1210372; the SN 2009ip research was also partly funded by NSF award AST-1211916.

\section{References}

Chornock, R., Filippenko, A. V., Li, W., \& Silverman, J. M. 2010, ApJ, 713, 1363

Davidson Jr., J. W., Bjorkman, K. S. Hoffman, J. L., et al. 2014, JAI, 03, 1450009

Dessart, L. \& Hillier, D. J. 2011, MNRAS, 415, 3497

Fraser, M., Magee, M., Kotak, R., et al. 2013, ApJ, 779, 8

Hoffman, J. L., Leonard, D. C., Chornock, R., et al. 2008, ApJ, 688, 1186

Hoffman, J. L., Smith, N., Bilinski, C., et al. 2014, AAS, 223, 35421 (http://goo.gl/hLOeYq)

Höflich, P. 1991, A\& A, 246, 481

Kasen, D., Nugent, P., Wang, L., et al. 2003, ApJ, 593, 788

Khandrika, H. G., Leonard, D. C., Horst, C., et al. 2014, AAS, 224, 121.16

Leonard, D. C., Filippenko, A. V., Barth, A. J., \& Matheson, T. 2000, ApJ, 536, 239

Leonard, D. C., Filippenko, A. V., Ganeshalingam, M., et al. 2006, Nature, 440, 505

Levesque, E. M., Stringfellow, G. S., Ginsburg, A. G., et al. 2014, AJ, 147, 23

Margutti, R., Milisavljevic, D., Soderberg, A. M., et al. 2014, ApJ, 780, 21

Mauerhan, J. C., Smith, N., Filippenko, A. V., et al. 2013, MNRAS, 430, 1801

Mauerhan, J., Williams, G. G., Smith, N., et al. 2014, MNRAS, 442, 1166

Mauerhan, J., Williams, G., Leonard, D. C., et al. 2015, MNRAS, 453, 4467

Porter, A. L., Leising, M. D., Williams, G. G., et al. 2016, ApJ, 828, 24

Ruiz-Lapuente, P. 2014, NewAR, 62, 15

Schmidt, G. D., Elston, R., \& Lupie, O. L. 1992, AJ, 104, 1563

Smartt, S. J. 2009, ARA\& $A, 47,63$

Wang, L. \& Wheeler, J. C. 2008, ARAA, 46, 433 